PАCS: 06.20.FN, 42.51. - P, 72.20, JV, 32.30.RJ; УДК 539.17

\title{
INTERPLAY OF THE HYPERFINE, ELECTROWEAK AND STRONG INTERACTIONS IN HEAVY HADRON-ATOMIC SYSTEMS AND X-RAY STANDARDS STATUS
}

\author{
T. N. Zelentsova, V. N. Pavlovich, I. N. Serga, N. V. Mudraya \\ Institute for Nuclear Researches, National Academy of Sciences of Ukraine, Kiev \\ Odessa National Polytechnical University, Odessa
}

\section{INTERPLAY OF THE HYPERFINE, ELECTROWEAK AND STRONG INTERACTIONS IN HEAVY HADRON-ATOMIC SYSTEMS AND X-RAY STANDARDS STATUS}

\section{T. N. Zelentsova, V. N. Pavlovich, I. N. Serga, N. V. Mudraya}

\begin{abstract}
It is given an analysis of the electromagnetic, hyperfine, electroweak and strong interactions contributions to the transitions energies in the X-ray spectra of hadronic atomic systems and the X-ray standards status. It is considered an advanced approach to redefinition of the mesonnucleon phenomenological optical model potential parameters and increasing an accuracy of the hadronic X-ray transitions energies definition.
\end{abstract}

Keywords: hyperfine, strong interactions, hadronic atomic systems, X-ray standards

\section{РОЛЬ НАДТОНКОЇ, ЕЛЕКТРОСЛАБКОЇ І СИЛЬНОЇ ВЗАЕМОДІЙ У АДРОН-АТОМНИХ СИСТЕМАХ ТА СТАТУС РЕНТГЕНІВСЬКИХ СТАНДАРТІВ}

\section{Т. М. Зеленцова, В. М. Павлович, І. М. Серга, Н. В. Мудра}

Анотація. Проаналізовані внески за рахунок надтонкої, електрослабкої, сильної взаємодій в енергії переходів у рентгенівських спектрах адрон-атомних систем і статус рентгенівських стандартів. Розглянуто ефективний підхід до перевизначення параметрів оптичного модельного мезон-нуклонного потенціалу і збільшенню точності визначення енергій переходів у рентгенівських спектрах адронних атомів.

\footnotetext{
Ключові слова: надтонка, сильна взаємодія, адронні атомні системи, рентгенівські стандарти
РОЛЬ СВЕРХТОНКОГО, ЭЛЕКТРОСЛАБОГО И СИЛЬНОГО ВЗАИМОДЕЙСТВИЙ В АДРОН- АТОМНЫХ СИСТЕМАХ И СТАТУС РЕНТГЕНОВСКИХ СТАНДАРТОВ

\section{Т. Н. Зеленцова, В. Н. Павлович, И. Н. Серга, Н. В. Мудрая}

Аннотация. Проанализированы вклады электромагнитного, сверхтонкого, электрослабого и сильного взаимодействий в энергии переходов в рентгеновских спектрах адрон-атомных систем и статус рентгеновских стандартов. Рассмотрен эффективный подход к переопределению параметров оптического модельного мезон-нуклонного потенциала и увеличению точности определения энергий переходов в рентгеновских спектрах адронных атомов.

Ключевые слова: сверхтонкое, сильное взаимодействие, адронные атомные системы, рентгеновские стандарты 
In this paper we give an analysis of the electromagnetic, hyperfine, electroweak and strong interactions contributions to the transitions energies in the X-ray spectra of hadronic atomic systems and the X-ray standards status. Besides, wt is considered an advanced approach to redefinition of the meson-nucleon phenomenological optical model potential parameters and increasing an accuracy of the hadronic X-ray transitions energies definition.

At present time the transition energies in the pionic and kaonic atoms are measured with an unprecedented precision and from studying spectra of the pionic and kaonic atoms (hydrogen, helium etc) it is possible to investigate the strong interaction at low energies by measuring the energy and natural width of the ground level with a precision of few meV [1-20]. Besides, some pionic atoms can additionally be used to define new low-energy Xray standards and to evaluate the pion mass using high accuracy X-ray spectroscopy [1-15]. Similar endeavour are in progress with kaonic atoms [2].

At present time several highly prŭcised measurements are carried out for the kaonic (pionic) hydrogen, helium and other elements. In the known experiments [11] at the Japan Synchrotron Centre the strong interaction shift of the $1 s$ state in kaonic hydrogen was found to be repulsive in agreement with kaon nucleus scattering data and in disagreement with the earlier kaonic hydrogen experiments. The origin of this repulsive strong interaction at threshold can be traced back to the presence of the (1405) resonance which leads on one hand to a repulsive $K$ - $p$ scattering length and on the other to the possible existence of strongly bound kaonic states in light nuclei [12]. Another impressive experiment DEAR ("Dapne exotic atoms research") allowed to perform an improved measurement of kaonic hydrogen [15] (look fig. 1). It has been received significantly higher precision and smaller shift and width values than the values of the experiment [11].

The similar experiments are performed or in a status of preparing for the pionic systems (look c.g. $[1,2,9,10])$. It is important to note that because of the tiny strong interaction effect the study of pionic hydrogen and other elements calls for an Xray spectrometer system with ultimate precision, provided by a crystal spectrometer which is feasible due to the huge pion beam intensity. In principle the isospin dependent pion-nucleon scattering lengths can be extracted from the measurement of the shift and width of pionic hydrogen alone using Deser-type formulae [1]. In the kaonic hydrogen case however the corresponding scattering length can only be determined by measuring the shift and width of kaonic hydrogen as well as kaonic deuterium.

In refs. $[1,2,9,10]$ is has been demonstrated that narrow lines from hydrogen-like pionic atoms are able to serve as energy standards in the few $\mathrm{keV}$ range. By using this method the energy uncertainty is limited primarily by the knowledge of the charged pion mass, whenever transitions close in energy can be found. One could remind that the widespread $\mathrm{X}$-ray energy standards are created by exciting inner-shell transitions in atoms with either electrons or photons. In a number of cases their energies are given with precision close to $1 \mathrm{ppm}$ [1-3], which does not necessarily mean that these standards can be used to such an accuracy. For X-rays originating from inner-shell transitions in multielectron systems, the center of gravity of the line cannot be attributed unambiguously to a physical transition. Besides, it is obvious that the transitions line shape in the multi-electron atomic systems is dependent upon the excitation mechanism used to create the inner-shell vacancies. Here it is worth to underline that the chemical environment of the atom can play a significant role [21]. This problem also affects transition energies, particularly when they involve M2,3 and N2,3 shells. An inherent problem with current standard X-ray lines is their natural width, which is typically more than 10 times larger than the resolution of the best X-ray spectrometers. Taking into account the above said, it is natural to use the exotic hadron-atoms and heavy ions sources as an alternative and more general approach to both gamma- rays or natural X-rays (photon emitters in the few keV range) Generally speaking, the muonic, kaonic and pionic atoms are the acceptable candidates, which provide a dense set of lines. It can be supplemented by an even denser set if one uses antiprotonic atoms with beam intensities comparable to the obtained ones at the European Centre of Nuclear Research CERN [1,2].

The most known theoretical models to treating the hadronic atomic systems are presented in refs. [9-11,16-21]. In refs. [16-20] there were developed the effective ab initio schemes to the Klein-Gordon equation solution and further definition of the $\mathrm{X}-$ ray spectra for multi-electron kaonic atoms with the different schemes for account for the nuclear, radiative, correlation effects. The theoretical studying the strong interaction shifts and widths from $\mathrm{X}$ ray spectroscopy of kaonic atoms ( $\mathrm{U}, \mathrm{Pb}$ etc) was 
fulfilled. The most difficult aspects of the theoretical modeling are reduced to the correct description of the kaon (pion)-strong interaction as the electromagnetic part of the problem is reasonably accounted for in models [16-19]. Besides, quite new aspect is linked with the possible, obviously, very tiny electroweak and hyperfine interactions.

All available theoretical models to treating the hadronic (kaonic, pionic) atoms are naturally based on the using the Klein-Gordon equation for kaon (pion) particle [5]:

$$
m^{2} c^{2} \Psi(x)=\left\{\frac{1}{c^{2}}\left[i \hbar \partial_{t}+e V_{0}(r)\right]^{2}+\hbar^{2} \nabla^{2}\right\} \Psi(x),
$$

where $\mathrm{c}$ is a speed of the light, $\hbar$ is the Planck constant, $e$ is the particle (here is equal the electron charge), $m$ is the particle mass and $\Psi_{0}(\mathrm{x})$ is the scalar wave function of the space-temporal coordinates. Usually one considers the central potential $\left[\mathrm{V}_{0}(\mathrm{r})\right.$, $0]$ approximation with the stationary solution:

$$
\Psi(\mathrm{x})=\exp (-\mathrm{iE} t / \hbar) \phi(x)
$$

where $\phi(x)$ is the solution of the stationary equation.

$$
\left\{\frac{1}{c^{2}}\left[E+e V_{0}(r)\right]^{2}+\hbar^{2} \nabla^{2}-m^{2} c^{2}\right\} \phi(x)=0
$$

Here $\mathrm{E}$ is the total energy of the system (sum of the mass energy $\mathrm{mc}^{2}$ and binding energy $\varepsilon_{0}$ ). In principle, the central potential $\mathrm{V}_{0}$ naturally includes the central Coulomb potential, the vacuum-polarization potential, the strong interaction potential. Standard approach to treating the last interaction is provided by the well known optical potential model (c.g. [11]). Practically in all works the central potential $\mathrm{V}_{0}$ is the sum of the following potentials. The nuclear potential for the spherically symmetric density $\rho(r \mid R)$ is:

$$
\begin{aligned}
V_{\text {nucl }}(r \mid R) & =-\left((1 / r) \int_{0}^{r} d r_{1} r_{1}^{2} \rho\left(r_{1} \mid R\right)+\right. \\
& +\int_{r}^{\infty} d r_{1} r_{1} \rho\left(r_{1} \mid R\right),
\end{aligned}
$$

where $R$ is a radius of the kaonic (pionic) atom. The most popular Fermi-model approximation the charge distribution in the nucleus $\rho(r)$ (c.f. $[1,2])$ is as follows:

$$
\left.\tilde{n}(r)=\tilde{n}_{0} /\{1+\exp [(r-c) / a)]\right\},
$$

where the parameter $a=0.523 \mathrm{fm}$, the parameter $c$ is chosen by such a way that it is true the following condition for average-squared radius: $\left\langle r^{2}\right\rangle^{1 / 2}=\left(0.836 \cdot A^{1 / 3}+0.5700\right) \mathrm{fm}$. The effective algorithm for its definition is used in refs. [7,18-20] and reduced to solution of the following system of the differential equations:

$$
\begin{gathered}
V^{\prime} \operatorname{nucl}(r, R)=\left(1 / r^{2}\right) \int_{0}^{r} d r_{1} r_{1}^{2} \rho\left(r_{1}, R\right) \equiv\left(1 / r^{2}\right) y(r, R) \\
y^{\prime}(r, R)=r^{2} \rho(r, R) \\
\left.\tilde{n}^{\prime}(r)=\left(\tilde{n}_{0} / a\right) \exp [(r-c) / a]\{1+\exp [(r-c) / a)]\right\}^{2}
\end{gathered}
$$

with the boundary conditions:

$$
\begin{gathered}
V_{\text {nucl }}(0, R)=-4 /(\pi r) \\
y(0, R)=0, \\
\tilde{n}(0)=\tilde{n}_{0} /\{1+\exp [-c / a]\}
\end{gathered}
$$

Another, probably, more consistent approach is in using the relativistic mean-field (RMF) model, which been designed as a renormalizable mesonfield theory for nuclear matter and finite nuclei [2326].To take into account the radiation corrections, namely, the effect of the vacuum polarization there are traditionally used the Ueling potential and its different modifications such as [1-7]. The most difficult aspect is an adequate account for the strong interaction. In a case of the kaonic atomic systems the most popular approach to treating the strong interaction between the nucleus and orbiting kaon is the phenomenological optical potential of model, such as $[1,2]$ :

$$
V_{N}=-\frac{2 \pi}{\mu}\left[1+\frac{M_{K}}{M_{N}}\right]\left[A_{K p} \rho_{p}(r)+A_{K n} \rho_{n}(r)\right],
$$

where $M_{K}$ and $M_{N}$ are the kaon and nucleon masses and $\mu$ is the kaon-nucleus reduced mass,

$\rho_{p}(r), \rho_{n}(r)$ are the proton and neutron densities in the nucleus and $A_{K p}, A_{K n}$ are the complex effective $K p$ and $K n$ scattering lengths. The known Batty approximation [11] reduces Eq.(10) to the next expression:

$$
V_{N}=-\frac{2 \pi}{\mu}\left[1+\frac{M_{K}}{M_{N}}\right][a \rho(r)],
$$

where the effective averaged $\mathrm{K}$-nucleon scattering length: $a=[(0.34 \pm 0.03)+\mathrm{i}(0.84 \pm 0.03)](\mathrm{fm})$.

The presented value of the length has been indeed chosen to describe the low and middle $\mathrm{Z}$ nuclei [11]. The disadvantage of the usually used approach is connected with approximate definition of the proton and neutron densities and using the effective averaged K-nucleon scattering length. 
In the pion-nucleon state interaction one should use the following pulse approximation expression for scattering amplitude of a pion on the " $\mathrm{i}$ " nucleon $[1,2]$ :

$$
f_{i}(r)=\left\{b_{0}^{\prime}+b_{1}^{\prime}(t \tau)+\left[c_{0}^{\prime}+c_{1}^{\prime}(t \tau)\right] k k^{\prime}\right\} \delta\left(r-r_{i}\right)
$$

Where $t$ and $\tau$ are the isospines of the pion and nucleon. The nucleon spin proportional terms of the kind $\sigma\left[k k^{\prime}\right]$ are omitted. The constants in (10) can be expressed through usual s-wave $\left(\alpha_{2 T}\right)$ and p-wave $\left(\alpha_{2 T, 2 J}\right)$ scattering length ( $T$ and $J$-isospin and spin of the system $\pi N$. The corresponding parameters in the Compton wave length $\lambda_{\pi}$ terms are as follows:

$$
\begin{gathered}
b_{0}^{\prime}=\left(\alpha_{1}+2 \alpha_{3}\right) / 3=-0.0017 \lambda_{\pi} . \\
b_{0}^{\prime}=\left(\alpha_{3}-\alpha_{1}\right) / 3=-0.086 \lambda_{\pi} . \\
c_{0}^{\prime}=\left(4 \alpha_{33}+2 \alpha_{13}+2 \alpha_{31}+\alpha_{11}\right) / 3=-0.208\left(\lambda_{\pi}\right)^{3} . \\
c_{1}^{\prime}=\left(2 \alpha_{33}-2 \alpha_{13}+\alpha_{31}-\alpha_{11}\right) / 3=-0.184\left(\lambda_{\pi}\right)^{3} .
\end{gathered}
$$

The scattering amplitude for pion on a nucleus is further received as a coherent sum of the $\pi N$-scattering lengths. In approximation of the only s-wave interaction the corresponding potential can be written in the form $[1,2]$ :

$$
\begin{gathered}
V_{N}(r)=-2 \pi \hbar^{2} \mu_{\pi}^{-1} \times \\
\times\left[Z A^{-1} a_{p}+(A-Z) A^{-1} a_{n}\right] \rho(r) .
\end{gathered}
$$

where $\mathrm{Z}$ - is the nuclear charge, $\mathrm{A}$ is the mass number, $\mu_{\pi}$ is the reduces pion mass. The s-wave lengths of the $\pi^{-1} p$-scattering $a_{p}=\left(2 \alpha_{1}+\alpha_{3}\right) / 3$ and $\pi^{-1} n$ scattering $a_{n}=\alpha_{3}$; scattering are introduced to Eq. (11). Because of the equality between $a_{n}=b_{0}^{\prime}+b_{1}^{\prime}$ and $a_{p}=b_{0}^{\prime}-b_{1}^{\prime}$ (with an opposite sign) the theoretical shift of the s-level with $T=0(A=2 Z)$ from Eq. (12) is much less than the observed shift. Thus, the more correct approximation must take into account the effects of the higher orders.

In whole the energy of the hadronic atom is represented as the sum:

$$
E \approx E_{K G}+E_{F S}+E_{V P}+E_{N} ;
$$

Here $E_{K G}$-is the energy of meson (say, kaon) in a nucleus $(Z, A)$ with the point-like charge (dominative contribution in (12)), $E_{F S}$ is the contribution due to the nucleus finite size effect, $E_{V P}$ is the radiation correction due to the vacuum-polarization effect, $E_{N}$ is the energy shift due to the strong interaction $V_{N}$. The last contribution can be defined from the experimental energy values as:

$$
E_{N}=E-\left(E_{K G}+E_{F S}+E_{V P}\right) .
$$

From the other side the strong meson-nucleus interaction contribution can be found from the solution of the Klein-Gordon equation with the corresponding meson-nucleon potential. In this case, this contribution $E_{N}$ is the function of the potentials (8)-(11) parameters.

Let us further to analyse some theoretical and experimental results and present some proposals on the further improvement of the available theoretical approaches. In table 1 there are listed the experimental and theoretical X-ray energies for kaonic hydrogen for the 2-1 transition (in $\mathrm{keV}$ ), taken from refs. [16-19]. The improved measurement data [15] are also presented in table 1 and fig.1. In tables 2,3 the data on the hyperfine transition energies in the kaonic and pionic nitrogen are listed (from. Refs. $[16,19,20])$.

Table 1 Calculated $\left(\mathrm{E}_{\mathrm{c}}\right)$ and measured $\left(\mathrm{E}_{\mathrm{m}}\right)$ kaonic $\mathrm{H}$ atom $\mathrm{X}$ ray energies (in $\mathrm{keV}$ ) [15-19]

\begin{tabular}{|c|c|c|c|c|c|}
\hline $\begin{array}{c}\text { Transi- } \\
\text { tion }\end{array}$ & $\begin{array}{c}\mathrm{E}_{\mathrm{c}} \\
{[1]}\end{array}$ & $\begin{array}{c}\mathrm{E}_{\mathrm{c}}, \\
{[20]}\end{array}$ & $\begin{array}{c}\mathrm{E}_{\mathrm{c}} \\
{[16]}\end{array}$ & $\begin{array}{c}\mathrm{E}_{\mathrm{m}} \\
{[17]}\end{array}$ & $\begin{array}{c}\mathrm{E}_{\mathrm{m}} \\
{[1,15]}\end{array}$ \\
\hline $\mathrm{H}, 2-1$ & 6.65 & 6.481 & 6.480 & $6,44(8)$ & $6.675(60)$ \\
6,482 & & & $6,96(9)$ \\
\hline
\end{tabular}

The importance of the presented energy parameters is provided not only its great role in the X-ray spectroscopy of the hadronic systems. The high accuracy of the theoretical modelling and new experimental results gives an important information about properties of the nuclei and hadrons (see below), in particular, masses, magnetic moments, spins and parity of some hadrons. Sensing the key parameters of the hyperfine, electromagnetic and strong interactions is of a great importance too.

In table 4 the theoretical $\left(\mathrm{E}_{\mathrm{c}}\right)$ and measured $\left(\mathrm{E}_{\mathrm{m}}\right)$ $\mathrm{X}$-ray energies for some kaonic atoms are listed (from refs. $[1,16,17,19])$. Two numbers in the fourth column are corresponding to data of the different experimental groups.

In whole there is observed a physically reasonable agreement between the theoretical and experimental data. It should be noted quite acceptable agreement between the theoretical and improved experimental data for the 2-1 X-ray transition energy of kaonic hydrogen (look table 1 and fig.1). The discrepancies are the source for the further improvement of the theoretical approaches. In table 5 we present the calculated $(\mathrm{C})$ and measured $(\mathrm{M})$ strong interaction shifts $\Delta \mathrm{E}$ and widths $\mathrm{G}$ (in $\mathrm{keV}$ ) for the kaonic atoms $\mathrm{X}$-ray transitions, taken from 


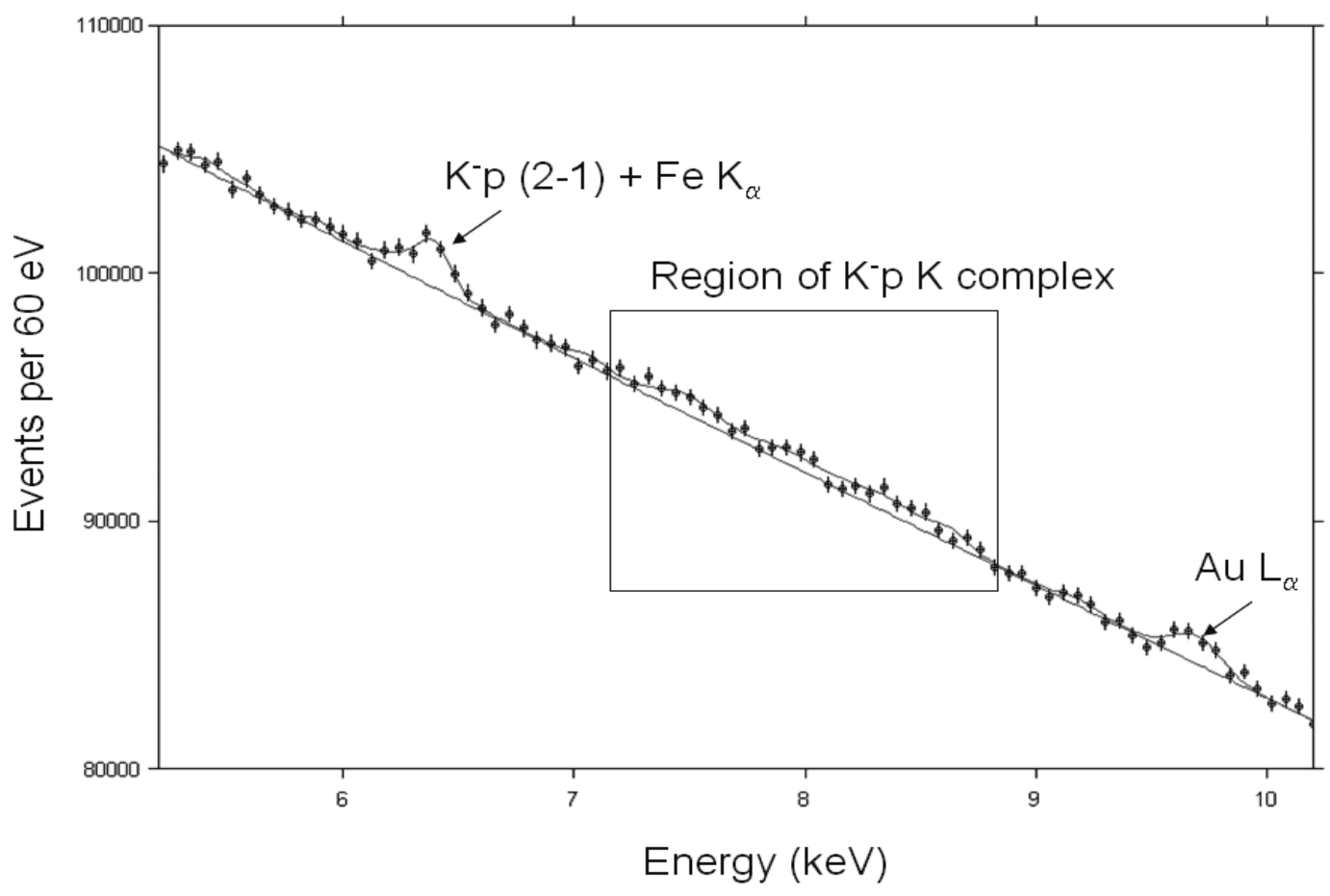

Fig. 1. The experimental kaonic hydrogen X-ray energy spectrum [15]

Hyperfine transition energies $(\mathrm{eV})$ in kaonic nitrogen $[16,19,20]$.

Table 2

\begin{tabular}{|c|c|c|c|c|c|c|}
\hline Transition & F-F' & $\begin{array}{c}\text { Trans. E (eV) } \\
{[16]}\end{array}$ & $\begin{array}{c}\text { Trans. E (eV) } \\
{[19]}\end{array}$ & Transition & F-F' & $\begin{array}{c}\text { Trans. E (eV) } \\
{[19]}\end{array}$ \\
\hline $8 \mathrm{k}-7 \mathrm{i}$ & $8-7$ & 2969.6365 & 2969.6289 & $8 \mathrm{i}-7 \mathrm{~h}$ & $7-6$ & 2970.4107 \\
\hline & $7-6$ & 2969.6383 & 2969.6298 & & $6-5$ & 2970.4135 \\
\hline & $7-7$ & 2969.6347 & 2969.6264 & & $6-6$ & 2970.4086 \\
\hline & $6-5$ & 2969.6398 & 2969.6345 & & $5-4$ & 2970.4193 \\
\hline & $6-6$ & 2969.6367 & 2969.6284 & & $5-5$ & 2970.4114 \\
\hline & $6-7$ & 2969.6332 & 2969.6248 & & $5-6$ & 2970.4073 \\
\hline
\end{tabular}

Hyperfine transition energies $(\mathrm{eV})$ in the pionic nitrogen $[16,19]$.

\begin{tabular}{|c|c|c|c|c|c|c|}
\hline Transition & F-F' & $\begin{array}{c}\text { Trans. E (eV) } \\
{[16]}\end{array}$ & $\begin{array}{c}\text { Trans. E (eV) } \\
{[19]}\end{array}$ & Transition & F-F' & $\begin{array}{c}\text { Trans. E (eV) } \\
{[19]}\end{array}$ \\
\hline $5 \mathrm{~g}-4 \mathrm{f}$ & $5-4$ & 4055.3779 & 4055.3744 & $5 \mathrm{f}-4 \mathrm{~d}$ & $4-3$ & 4057.6821 \\
\hline & $4-3$ & 4055.3821 & 4055.3784 & & $3-2$ & 4057.6914 \\
\hline & $4-4$ & 4055.3762 & 4055.3735 & & $3-3$ & 4057.6793 \\
\hline & $3-2$ & 4055.3852 & 4055.3828 & & $2-1$ & 4057.6954 \\
\hline & $3-3$ & 4055.3807 & 4055.3769 & & $2-2$ & 4057.6892 \\
\hline & $3-4$ & 4055.3747 & 4055.3712 & & $2-3$ & 4057.6768 \\
\hline
\end{tabular}

Calculated $\left(\mathrm{E}_{\mathrm{c}}\right)$ and measured $\left(\mathrm{E}_{\mathrm{m}}\right)$ kaonic atoms $\mathrm{X}$-ray energies (in keV)

\begin{tabular}{|c|c|c|c|c|c|c|}
\hline Nucl. & Transition & $\mathrm{E}_{\mathrm{c}}[1]$ & $\mathrm{E}_{\mathrm{c}}[16]$ & $\mathrm{E}_{\mathrm{c}},[19]$ & $\mathrm{E}_{\mathrm{c}},[17]$ & $\mathrm{E}_{\mathrm{m}}$ \\
\hline $\mathrm{W}$ & $8-7$ & - & - & 346.572 & 346.54 & $346.624(25)$ \\
\hline $\mathrm{W}$ & $7-6$ & - & - & 535.136 & 535.24 & $534.886(92)$ \\
\hline $\mathrm{U}$ & $8-7$ & 538.013 & 537.44 & 538.528 & 535.24 & $538.315(100)$ \\
\hline
\end{tabular}


Table 5

Calculated (C) and measured ( $\mathrm{M}$ ) strong interaction shifts $\Delta \mathrm{E}$ and widths $\mathrm{G}$ for $\mathrm{K}^{-}$-atoms $\mathrm{X}$-ray transitions: a- shift was estimated with Miller et al measured energy [1]; b - shift estimated with Cheng et al measured energy [2]; $\mathrm{c}-$ theory [11]; $\mathrm{d}$ - theory [20].

\begin{tabular}{|c|c|c|c|c|c|c|}
\hline Nucl & $\Delta \mathrm{E}_{\mathrm{C}}(\mathrm{d})$ & $\mathrm{G}_{\mathrm{C}}(\mathrm{d})$ & $\Delta \mathrm{E}_{\mathrm{C}}(\mathrm{c})$ & $\mathrm{G}_{\mathrm{C}}(\mathrm{c})$ & $\Delta \mathrm{E}_{\mathrm{M}}$ & $\mathrm{G}_{\mathrm{M}}$ \\
\hline $\begin{array}{c}\mathrm{W}, \\
8-7\end{array}$ & 0.038 & 0.072 & -0.003 & 0.065 & $\begin{array}{c}0.079^{\mathrm{c}} \\
0.052^{\mathrm{d}}\end{array}$ & $0.070(15)$ \\
\hline $\begin{array}{c}\mathrm{W}, \\
7-6\end{array}$ & -0.294 & 3.85 & -0.967 & 4.187 & $\begin{array}{c}-0.353^{\mathrm{c}} \\
-0.250^{\mathrm{d}}\end{array}$ & $3.72(35)$ \\
\hline $\begin{array}{c}\mathrm{Pb}, \\
8-7\end{array}$ & 0.046 & 0.281 & -0.023 & 0.271 & $\begin{array}{c}0.072^{\mathrm{c}} \\
0.047^{\mathrm{d}}\end{array}$ & $\begin{array}{c}0.284(14) \\
0.370(150)^{\mathrm{a}}\end{array}$ \\
\hline $\begin{array}{c}\mathrm{U}, \\
8-7\end{array}$ & -0.205 & 2.620 & -0.189 & 2.531 & $\begin{array}{c}0.120^{\mathrm{a}} ; 0.032^{\mathrm{b}} \\
-0.40^{\mathrm{c}} ;-0.213^{\mathrm{d}}\end{array}$ & $\begin{array}{c}2.67(10) \\
1.50(75)^{\mathrm{a}}\end{array}$ \\
\hline
\end{tabular}

ref. $[1,2,11,16,20]$. Again several numbers in some columns are corresponding to data of the different experimental groups. The width $\mathrm{G}$ is the strong width of the lower level which was obtained by subtracting the electromagnetic widths of the upper and lower level from the measured value. The shift $\Delta \mathrm{E}$ is defined as difference between the measured $E_{M}$ and calculated $E_{E M}$ (electromagnetic) values of transition energies. The calculated value is obtained by direct solving the equation (3) with the optical model kaon-nucleon potential.

It is easily to understand that when there is the close agreement between theoretical and experimental shifts, the corresponding energy levels are not significantly sensitive to strong nuclear interaction, i.e the electromagnetic contribution is dominative. In the opposite situation the strong-interaction effect is very significant. The detailed analysis of theoretical and separated experimental data shows that indeed there is a physically reasonable agreement between the cited data. But, obviously, there may take a place the exception too as it is shown on example of the kaonic uranium. Further one can perform the comparison of the theoretically and experimentally defined transition energies in the $\mathrm{X}$-ray spectra and further make redefinition of the meson-nucleon model potential parameters using Eqs. (8)-(11). Taking into account the increasing accuracy of the X-ray hadronic atoms spectroscopy experiments, one can conclude that the such a way will make more clear the true values for parameters of the kaon (pion)-nuclear potentials and correct the disadvantage of widely used parameterization of the potentials (8)-(11) from the light nuclei physics. Besides, the known perspective can be opened on the way of sensing the parity non-conservation in the heavy hadron multi-electron atomic systems. Obviously, this effect will be small in the light hadronic systems as $\mathrm{K}^{-}-\mathrm{H}, \mathrm{K}^{-}-\mathrm{N}$, but its contribution is increasing as $Z^{3}$, so one could wait for the increased contribution in the high- $\mathrm{Z}$ atoms such as $\mathrm{K}^{-}-\mathrm{Pb}$, $\mathrm{K}^{-}-\mathrm{Vi}, \mathrm{K}^{-} \mathrm{U}$ etc.

Acknowledgements. Authors would like to thank Prof. V. D. Rusov, Prof. A. V. Glushkov for useful discussions. The referee's comments are acknowledged too.

\section{References}

1. Deslattes R., Kessler E., Indelicato P., de Billy L., Lindroth E. , Anton J., Exotic atoms//Rev. Mod. Phys. - 2003. - Vol.75. - P.35-70.

2. Deloff A., Fundamentals in Hadronic Atom Theory, Singapore: World Sci., 2003. - 352P.

3. Glushkov A. V., Relativistic Quantum Theory. Quantum, mechanics of Atomic Systems. - Odessa: Astroprint, 2008. - 900P.

4. Hayano R. S., Hori M., Horvath D., Widman E., Antiprotonic helium and CPT invariance//Rep. Prog. Phys. - 2007. - Vol.70. - P.1995-2065.

5. Mohr P. J. Quantum Electrodynamics Calculations in few-Electron Systems// Phys. Scripta. - 1993. Vol.46,N1. - P.44-52.

6. Quiney H. M., Grant I. P. Partial-wave mass renormalization in atomic QED calculation // Phys.Scripta T. - 1993. - Vol.46. - P.132-138.

7. Glushkov A. V., Rusov V. D., Ambrosov S. V., Loboda A. V., Resonance states of compound super-heavy nucleus and EPPP in heavy nucleus collisions // New Projects and New Lines of research in Nuclear physics.Eds. Fazio G. and Hanappe F.: Singapore, World Sci. - 2003. - P. 142-154.

8. Gall K. P., Austin E., Miller J. P., et al, Precision measurements of $\mathrm{K}^{-}$and $\Sigma^{-}$masses//Phys.Rev.Lett. 1988. - Vol.60. - P.186-190.

9. Anagnostopoulos D. F., Biri S., Boisbourdain V., Demeter M., Borchert G., Egger J., Fuhrmann H., Gotta D., Gruber A., Hennebach M. et al// Lowenergy X-ray standards from pionic atoms//Nucl. Instrum. Methods B. - 2003. - Vol.205. - P.918. 
10. Anagnostopoulos D. F. , Gotta D., Indelicato P., Simons L. M., Low-energy X-ray standards from hydrogenlike pionic atoms//Arxiv: phys. - 2003. 0312090v1-P.1-4.

11. Batty C. J., Eckhause M., Gall K. P., et al. Strong interaction effects in high $\mathrm{Z}^{-} \mathrm{K}^{-}$atoms//Phys. Rev. C. - 1989. - Vol.40. - P.2154-2160.

12. S. Okada, G. Beer, H. Bhang, M. Cargnelli, J. Chiba, Seonho Choi, C. Curceanu, Y. Fukuda, T. Hanaki, R. S. Hayano, M. Iio, T. Ishikawa, S. Ishimoto, T. Ishiwatari, K. Itahashi, M. Iwai, M. Iwasaki, B. Juhasz, P. Kienle, J. Marton, Y. Matsuda, H. Ohnishi, H. Outa, M. Sato, P. Schmid, S. Suzuki, T. Suzuki, H. Tatsuno, D. Tomono, E. Widmann, T. Yamazaki, H. Yimc and J. Zmeskal, Precision measurement of the $3 \mathrm{~d}->2 \mathrm{p}$ x-ray energy in kaonic ${ }^{4} \mathrm{He} / /$ Phys. Lett.B. - 2007. - Vol.653, N 5-6. - P. 387-391.

13. Ito T. M., Hayano R. S., Nakamura S. N., Terada T. P., Observation of kaonic hydrogen atom x rays// Phys. Rev. C. - 1998. - Vol.58. - P.2366 - 2382

14. Ishiwatari T. on behalf of the SIDDHARTA Collaboration, Silicon drift detectors for the kaonic atom Xray measurements in the SIDDHARTA experiment// Nucl.Instr. and Methods in Physics. Research Sec.A. Accelerators, Spectrometers, Detectors and Associated Equipment. - 2007. - Vol.581,N1-2. - P.326329.

15. M. Cargnelli, T. Ishiwatari, P. Kienle, J. Marton, E. Widmann, J. Zmeskal, G. Beer, A. M. Bragadireanu, T. Ponta, M. Bazzi, M. Catitti, C. Curceanu (Petrascu), C. Guaraldo, M. Iliescu, P. Levi Sandri, V. Lucherini, D. Pietreanu, D. L. Sirghi, F. Sirghi, P. Lechner, H. Soltau, L. Bombelli, C. Fiorini, T. Frizzi, A. Longoni, F. Ghio, B. Girolami, and L. Struder, Kaonic hydrogen $\mathrm{X}$ rays - experiments at DAFNE// Proc. KAON Int. Conf., Laboratori Nazionali di Frascati dell'INFN (Rome, Italy). 2007. - 5P.

16. Santos J. P., Parente F., Boucard S., Indelicato P., Desclaux j.P., X-ray energies of circular transitions and electron scattering in kaonic atoms//Phys. Rev.A. - 2005. - Vol.71. - P.032501.
17. Indelicato P., Trassinelli M., From heavy ions to exotic atoms// arXiv:physics. - 2005. - V10510126v1. - 16P.

18. Sukharev D. E., Khetselius O.Yu., Dubrovskaya Yu.V., Sensing strong interaction effects in spectroscopy of hadronic atoms// Sensor Electr. and Microsyst. Techn. - 2009. - N3. - P.16-21.

19. Sukharev D. E., Florko T. A., Khetselius O.Yu., Dubrovskaya Yu.V., Bremsstrahlung and X-ray spectra for kaonic and pionic hydrogen and nitrogen//Photoelectr. - 2009. - N18. - P.16-20.

20. Sukharev D. E., Khetselius O.Yu., Turin A. V., Florko T. A., Estimating of X-ray spectra for kaonic atoms as tool for sensing the nuclear structure// Sensor Electr. and Microsyst. Techn. - 2009. - N1. P.30-35.

21. Glushkov A. V., Khetselius O.Yu., Gurnitskaya E. P., Loboda A. V., Sukharev D. E., Relativistic quantum chemistry of heavy ions and hadronic atomic systems: Spectra and energy shifts// Theory and Applications of Computational Chemistry (AIP). - 2009. Vol.1. - P.131-134.

22. Glushkov A. V., Khetselius O.Yu., Lovett L., Electron$\beta$-Nuclear Spectroscopy of Atoms and Molecules and Chemical Environment Effect on the $\beta$-Decay parameters// Advances in the Theory of Atomic and Molecular Systems Series: Progress in Theoretical Chemistry and Physics, Eds. Piecuch P., Maruani J., Delgado-Barrio G., Wilson S. (Berlin, Springer). 2009. - Vol. 20. - P. 125-172.

23. Serot B. D., Walecka J. D. , Advances in Nuclear Physics Vol. 16: The Relativistic Nuclear Many Body Problem. Plenum Press, New York, 1986.

24. Ring P, Schuck P, The Nuclear Many-Body Problem. - Berlin: Springer, 2000.

25. Gambhir Y. K., Bhagwat A., The relativistic mean field and some of its recent applications// Physics of Elem. Part. and Atom.Nucl. - 2006. - Vol.37. - P.366-436.

26. Khetselius O.Yu., Relativistic perturbation theory calculation of the hyperfine structure parameters for some heavy-element isotopes//Int. Journ. of Quantum Chemistry. - 2009. - Vol.109. - P. 3330-3335. 